\title{
Distinct Electronic Effects on Reductive Eliminations of Symmetric and Dissymmetric bis-Aryl Platinum Complexes
}

Shashank Shekhar and John F. Hartwig*

Contribution from the Department of Chemistry, Yale University, P.O. Box 208107, New Haven, Connecticut 06520-8107 E-mail: John.Hartwig@yale.edu

\section{Supplementary Material}

Figure S1. Hammett plot for the rates of reductive elimination from (DPPF)Pt $\left(\mathrm{C}_{6} \mathrm{H}_{4}-4-\mathrm{CH}_{3}\right)\left(\mathrm{C}_{6} \mathrm{H}_{4}-4-\mathrm{X}\right)$.

Figure S2. Hammett plot for the rates of reductive elimination from (DPPF)Pt $\left(\mathrm{C}_{6} \mathrm{H}_{4}-4-\mathrm{OMe}\right)\left(\mathrm{C}_{6} \mathrm{H}_{4}-4-\mathrm{X}\right)$.

Figure S3. $1 \mathrm{H}$ NMR spectrum of (DPPF) Pt $\left(\mathrm{C}_{6} \mathrm{H}_{4}-4-\mathrm{NMe}_{2}\right)_{2}$.

Figure S4. 31P NMR spectrum of (DPPF)Pt $\left(\mathrm{C}_{6} \mathrm{H}_{4}-4-\mathrm{NMe}_{2}\right)_{2}$.

Figure S5. ${ }^{1} \mathrm{H}$ NMR spectrum of (DPPF)Pt $\left(\mathrm{C}_{6} \mathrm{H}_{4}-4-\mathrm{CH}_{3}\right)\left(\mathrm{C}_{6} \mathrm{H}_{4}-4-\mathrm{NMe}_{2}\right)$.

Figure S6. ${ }^{31} \mathrm{P}$ NMR spectrum of (DPPF) Pt $\left(\mathrm{C}_{6} \mathrm{H}_{4}-4-\mathrm{CH}_{3}\right)\left(\mathrm{C}_{6} \mathrm{H}_{4}-4-\mathrm{NMe}_{2}\right)$.

Figure S7. ${ }^{1} \mathrm{H}$ NMR spectrum of (DPPF)Pt $\left(\mathrm{C}_{6} \mathrm{H}_{4}-4-\mathrm{OMe}\right)\left(\mathrm{C}_{6} \mathrm{H}_{4}-4-\mathrm{NMe}_{2}\right)$.

Figure S8. ${ }^{31} \mathrm{P}$ NMR spectrum of (DPPF) Pt $\left(\mathrm{C}_{6} \mathrm{H}_{4}-4-\mathrm{OMe}\right)\left(\mathrm{C}_{6} \mathrm{H}_{4}-4-\mathrm{NMe}_{2}\right)$.

Figure S9. ${ }^{1} \mathrm{H}$ NMR spectrum of (DPPF)Pt $\left(\mathrm{C}_{6} \mathrm{H}_{5}\right)\left(\mathrm{C}_{6} \mathrm{H}_{4}-4-\mathrm{NMe}_{2}\right)$.

Figure S10. ${ }^{31} \mathrm{P}$ NMR spectrum of (DPPF) Pt $\left(\mathrm{C}_{6} \mathrm{H}_{5}\right)\left(\mathrm{C}_{6} \mathrm{H}_{4}-4-\mathrm{NMe}_{2}\right)$.

Figure S11. ${ }^{1} \mathrm{H}$ NMR spectrum of (DPPF) Pt $\left(\mathrm{C}_{6} \mathrm{H}_{4}-4-\mathrm{Cl}\right)\left(\mathrm{C}_{6} \mathrm{H}_{4}-4-\mathrm{NMe}_{2}\right)$.

Figure S12. ${ }^{31} \mathrm{P}$ NMR spectrum of (DPPF)Pt $\left(\mathrm{C}_{6} \mathrm{H}_{4}-4-\mathrm{Cl}\right)\left(\mathrm{C}_{6} \mathrm{H}_{4}-4-\mathrm{NMe}_{2}\right)$.

Figure S13. ${ }^{1} \mathrm{H}$ NMR spectrum of (DPPF) Pt $\left(\mathrm{C}_{6} \mathrm{H}_{4}-4-\mathrm{CF}_{3}\right)\left(\mathrm{C}_{6} \mathrm{H}_{4}-4-\mathrm{NMe}_{2}\right)$.

Figure S14. ${ }^{31} \mathrm{P}$ NMR spectrum of (DPPF) Pt $\left(\mathrm{C}_{6} \mathrm{H}_{4}-4-\mathrm{CF}_{3}\right)\left(\mathrm{C}_{6} \mathrm{H}_{4}-4-\mathrm{NMe}_{2}\right)$.

Figure S15. A representative plot for the decay of bis-aryl platinum complexes. 


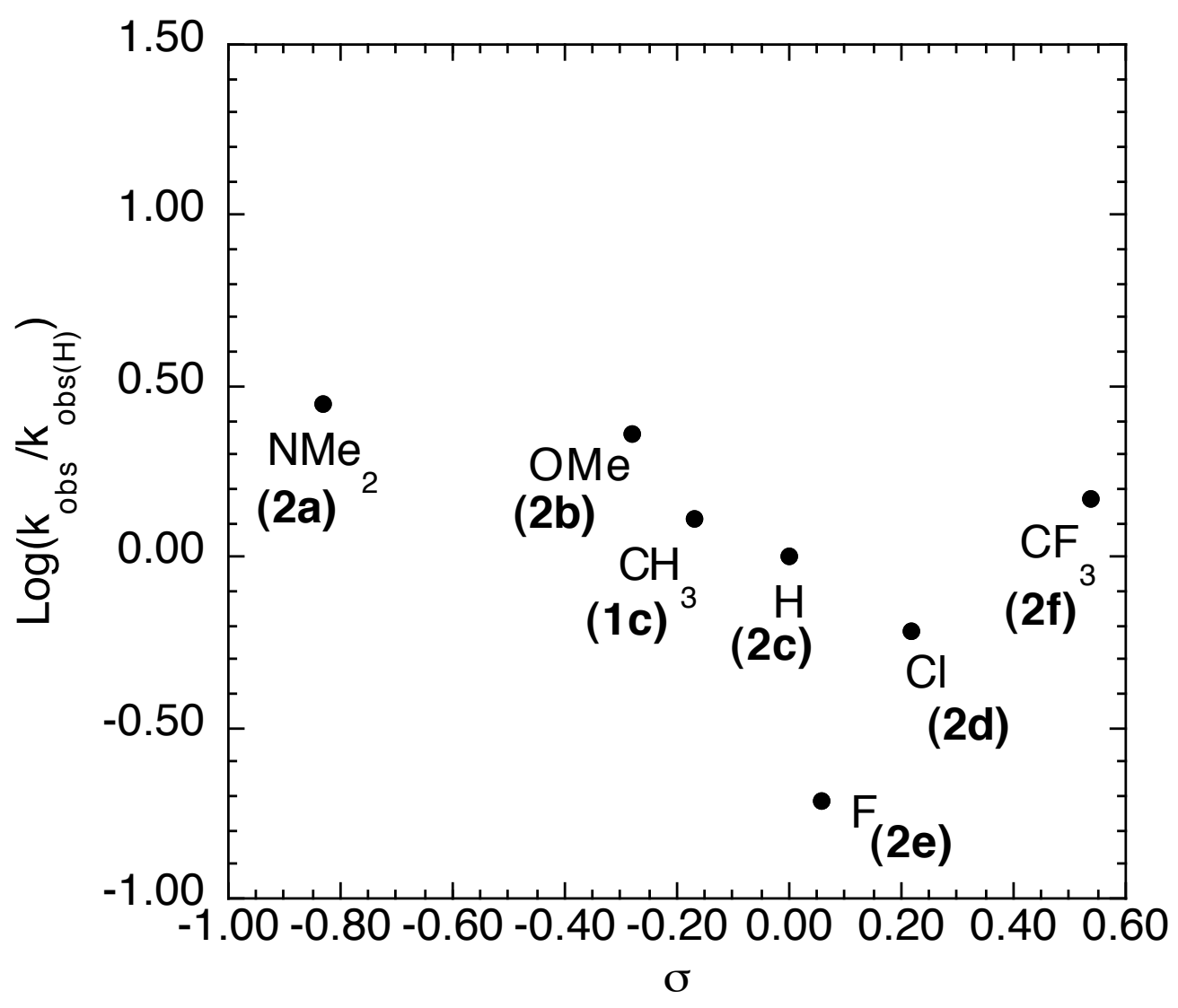

Figure S1. Hammett plot for the rates of reductive elimination reactions from (DPPF)Pt $\left(\mathrm{C}_{6} \mathrm{H}_{4}-4-\mathrm{CH}_{3}\right)\left(\mathrm{C}_{6} \mathrm{H}_{4}-4-\mathrm{X}\right)(\mathbf{1 b}, \mathbf{3 a - f})$. 


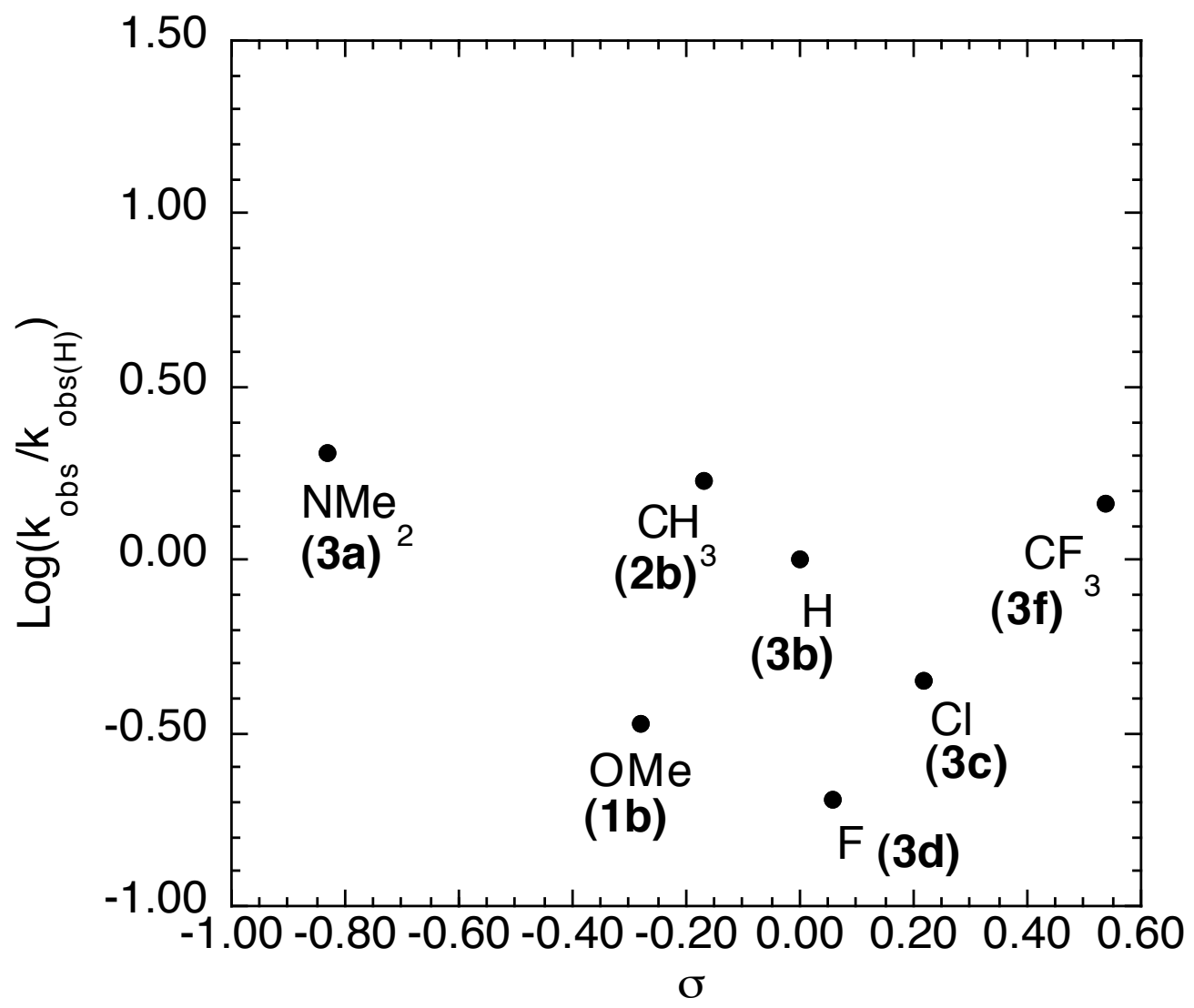

Figure S2. Hammett plot for the rates of reductive elimination reactions from (DPPF)Pt $\left(\mathrm{C}_{6} \mathrm{H}_{4}-4-\mathrm{OMe}\right)\left(\mathrm{C}_{6} \mathrm{H}_{4}-4-\mathrm{X}\right)(\mathbf{1 b}, \mathbf{2 b}, \mathbf{3 a - f})$. 


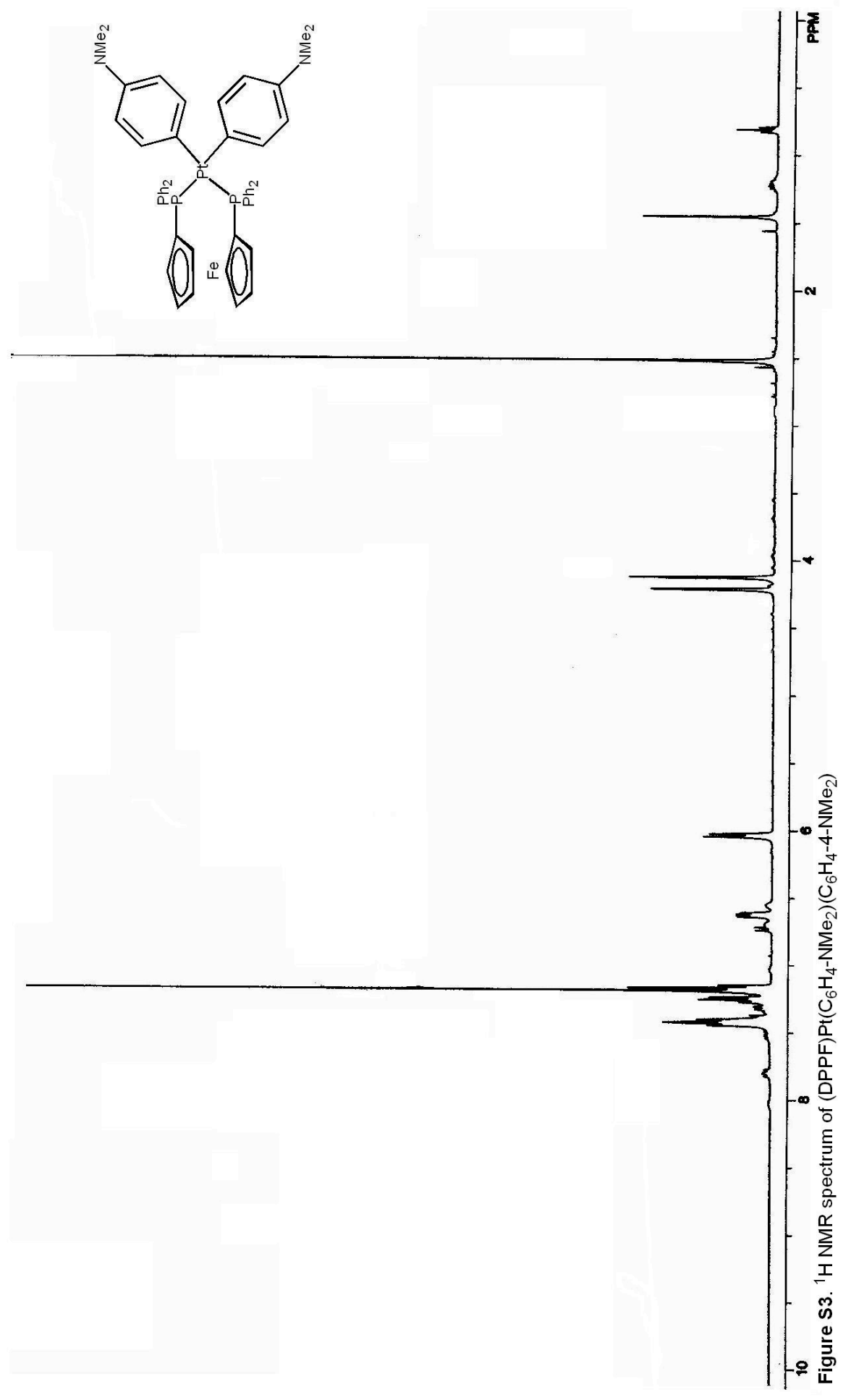



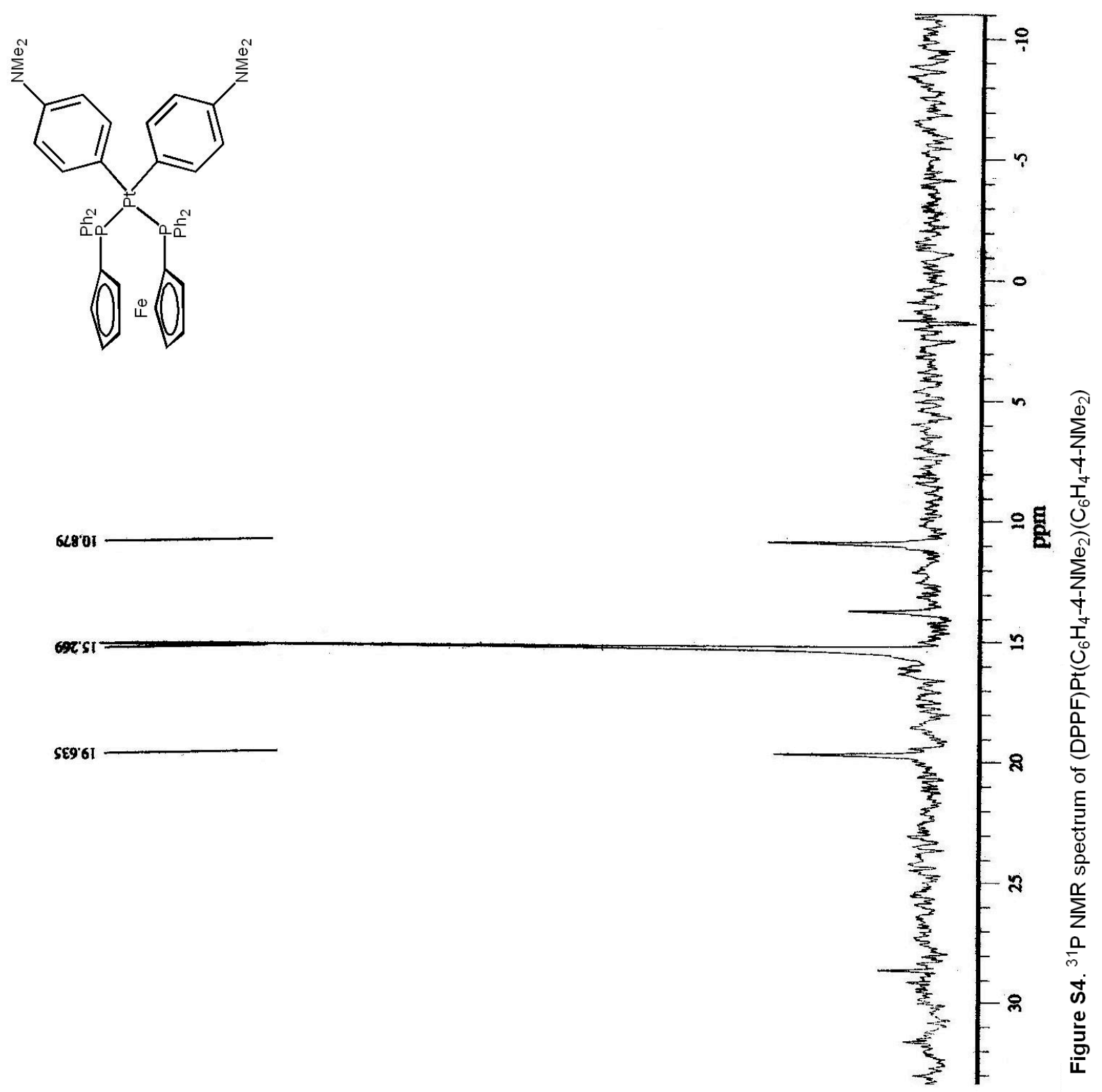

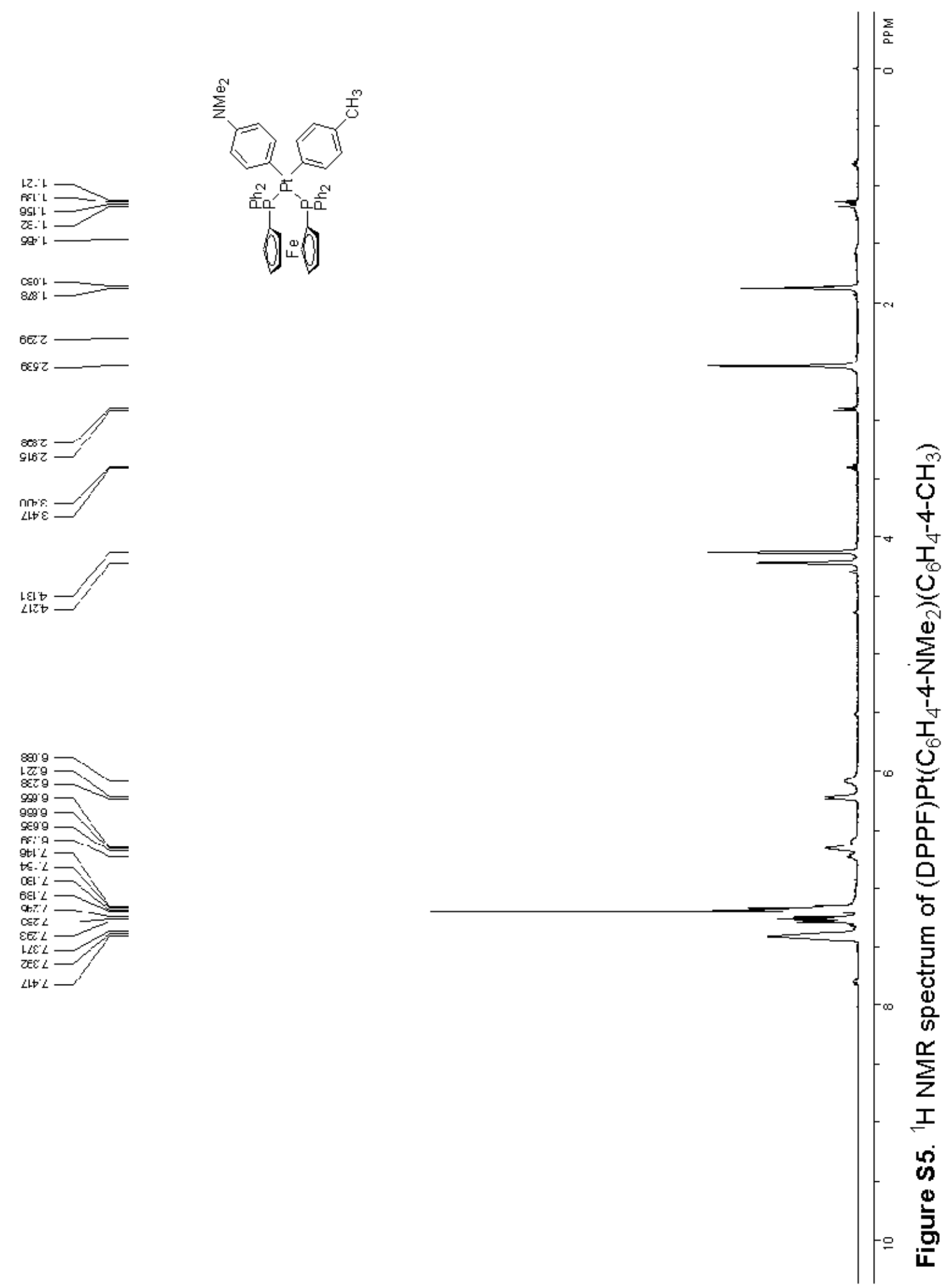


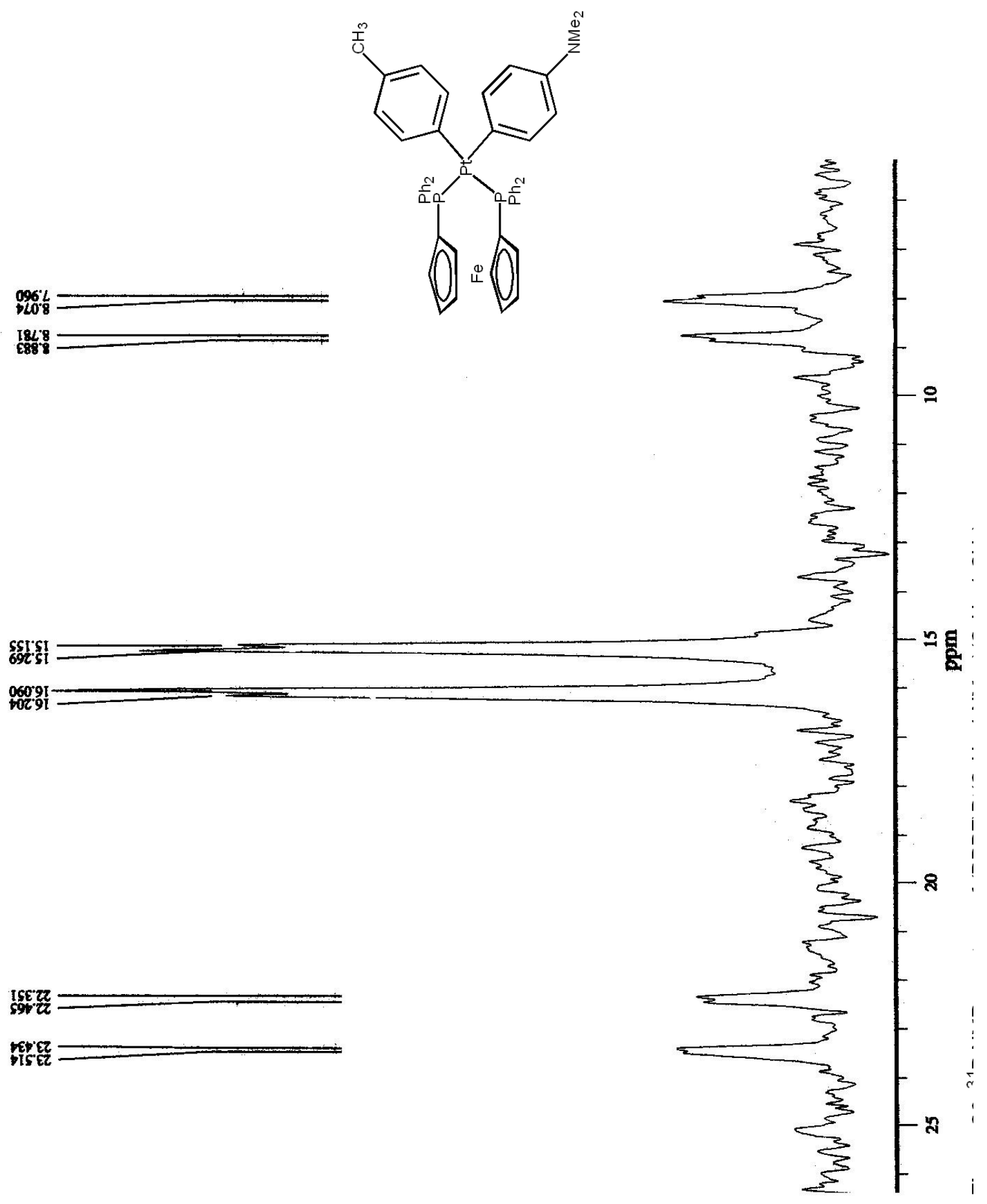




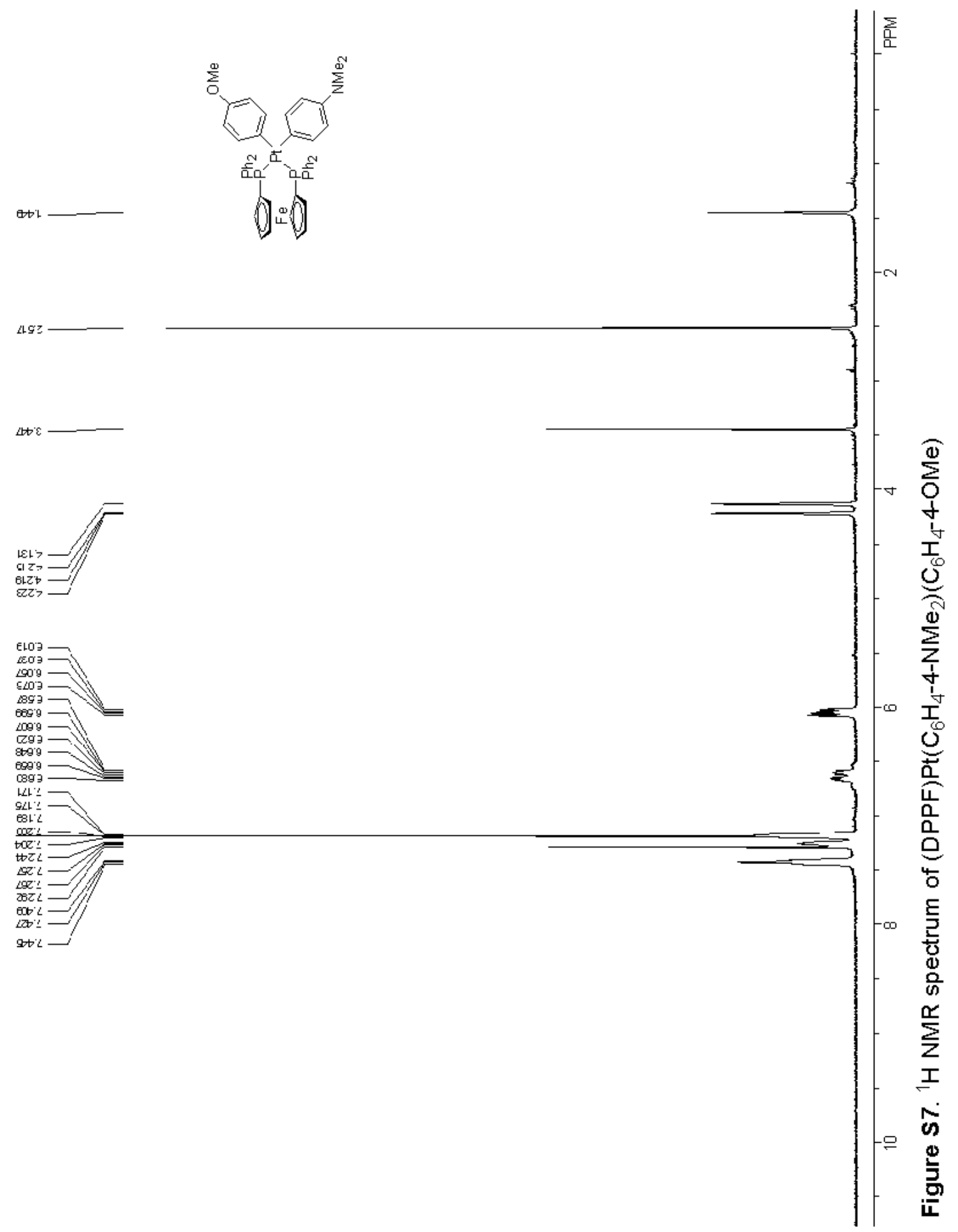


S9

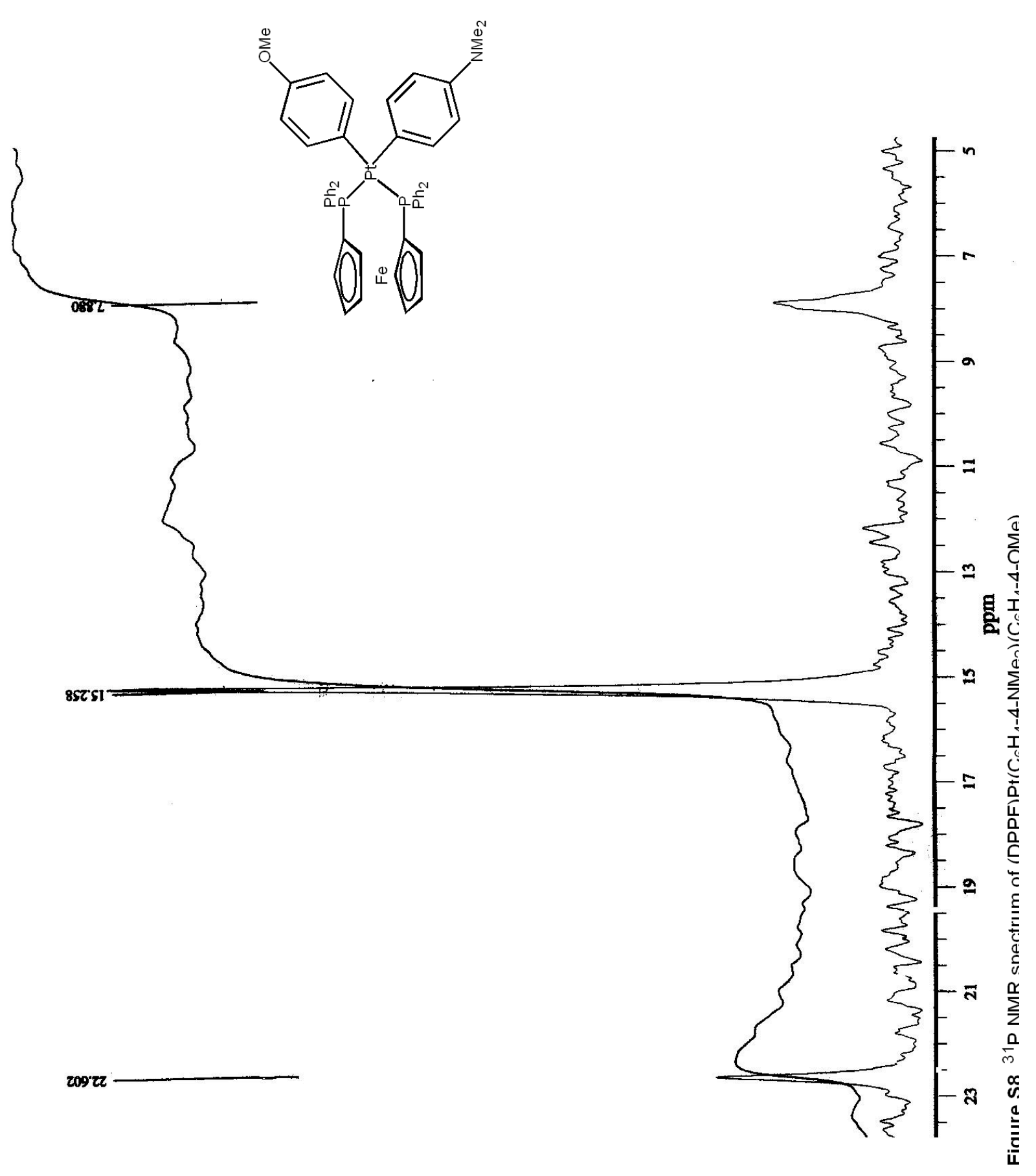



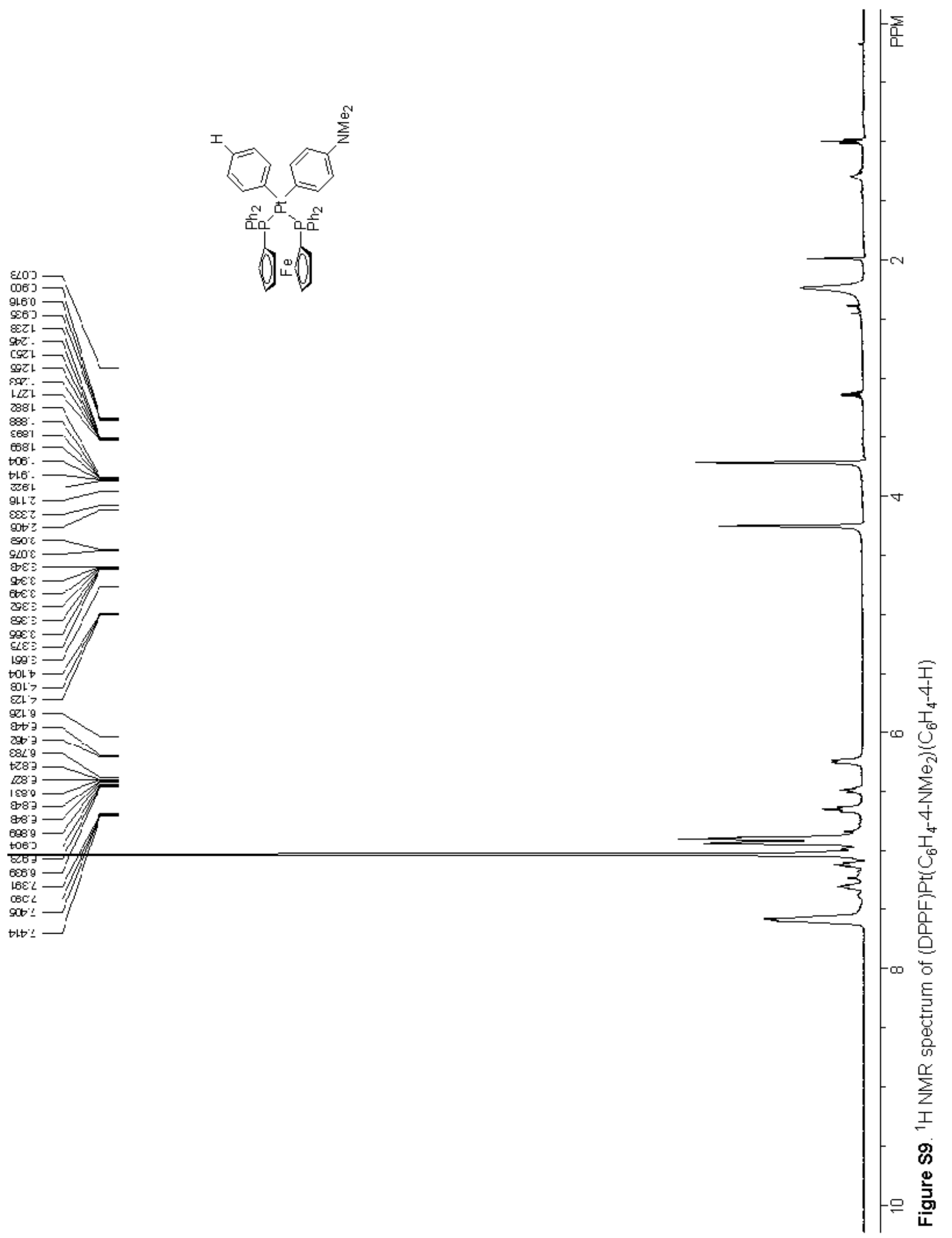

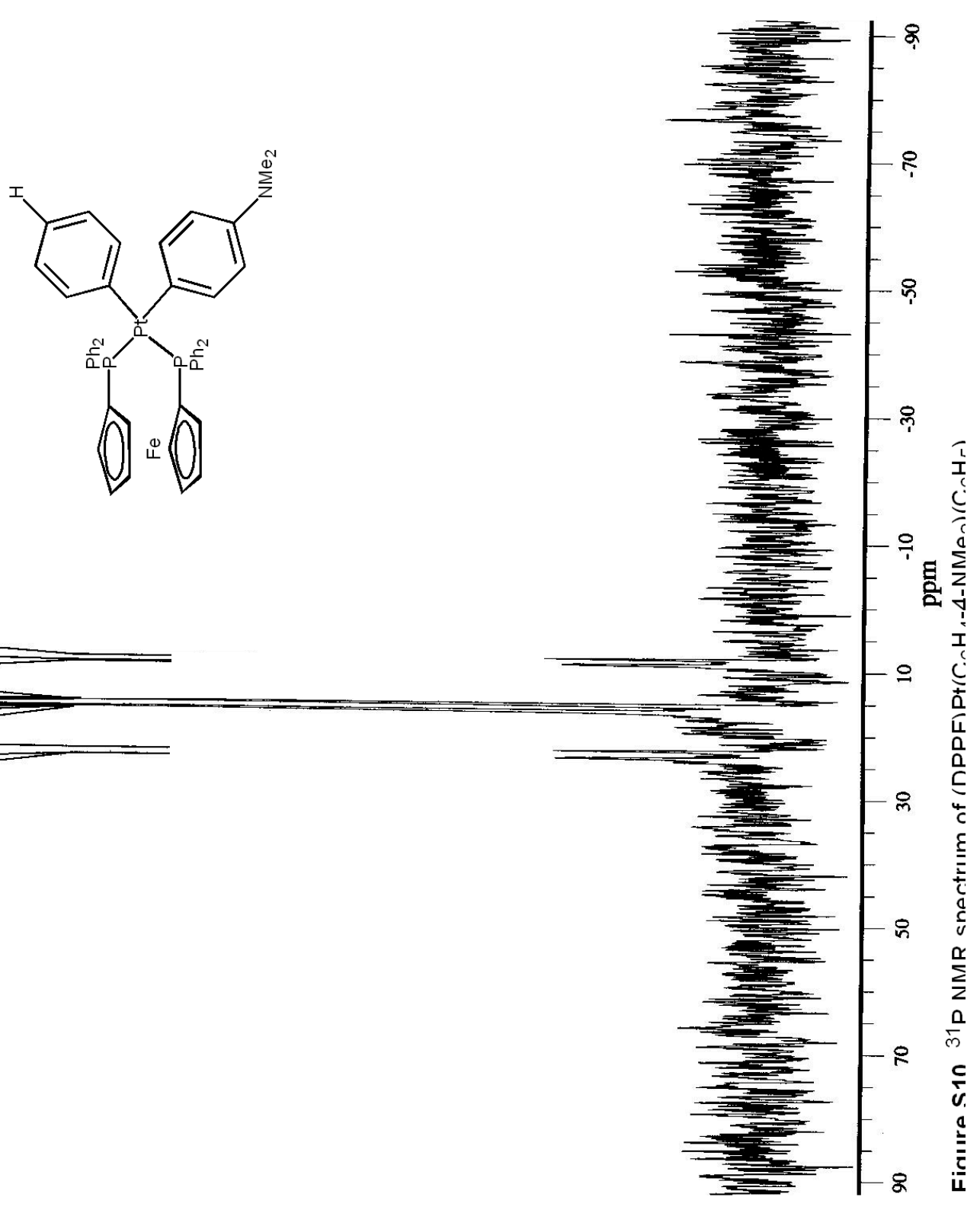

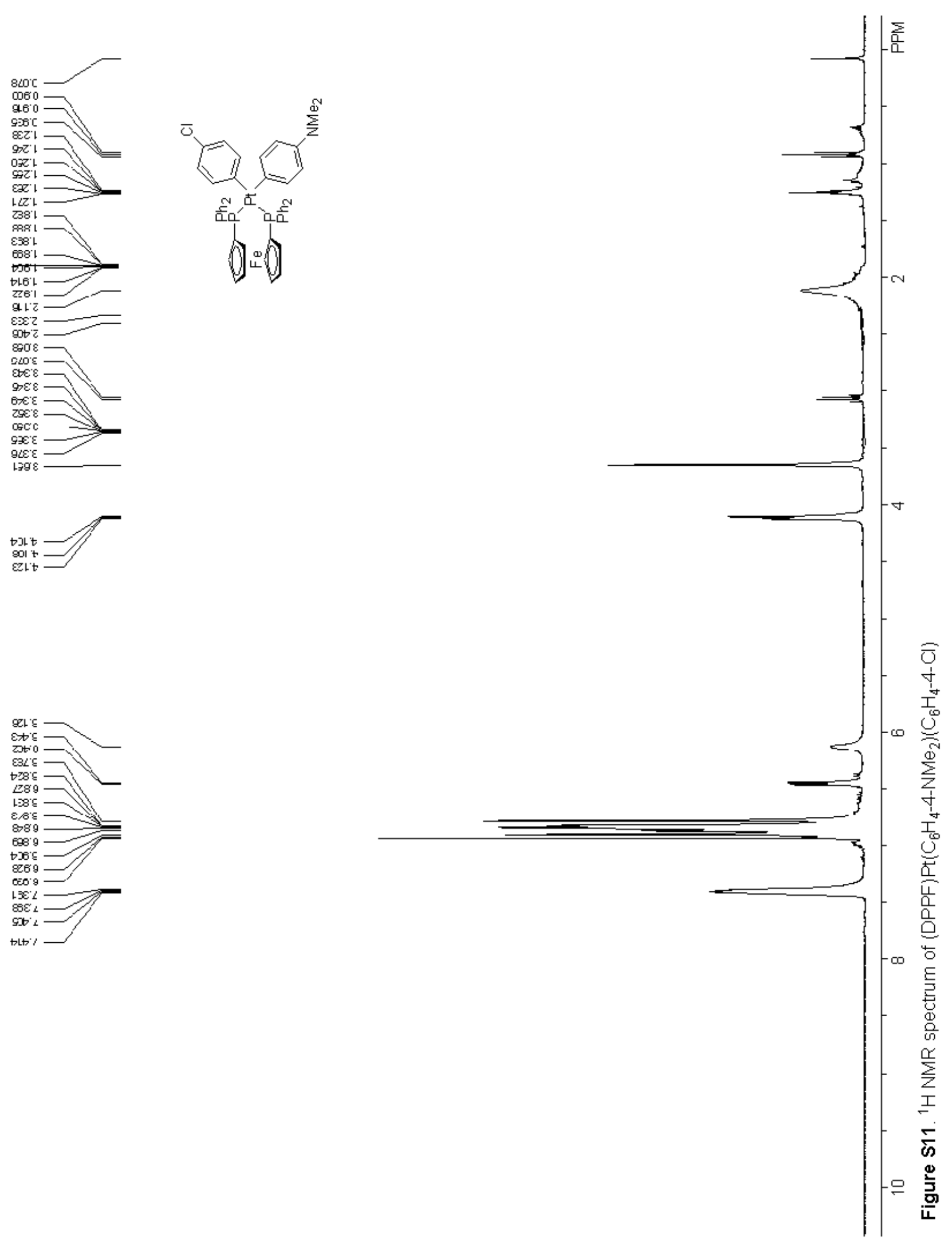

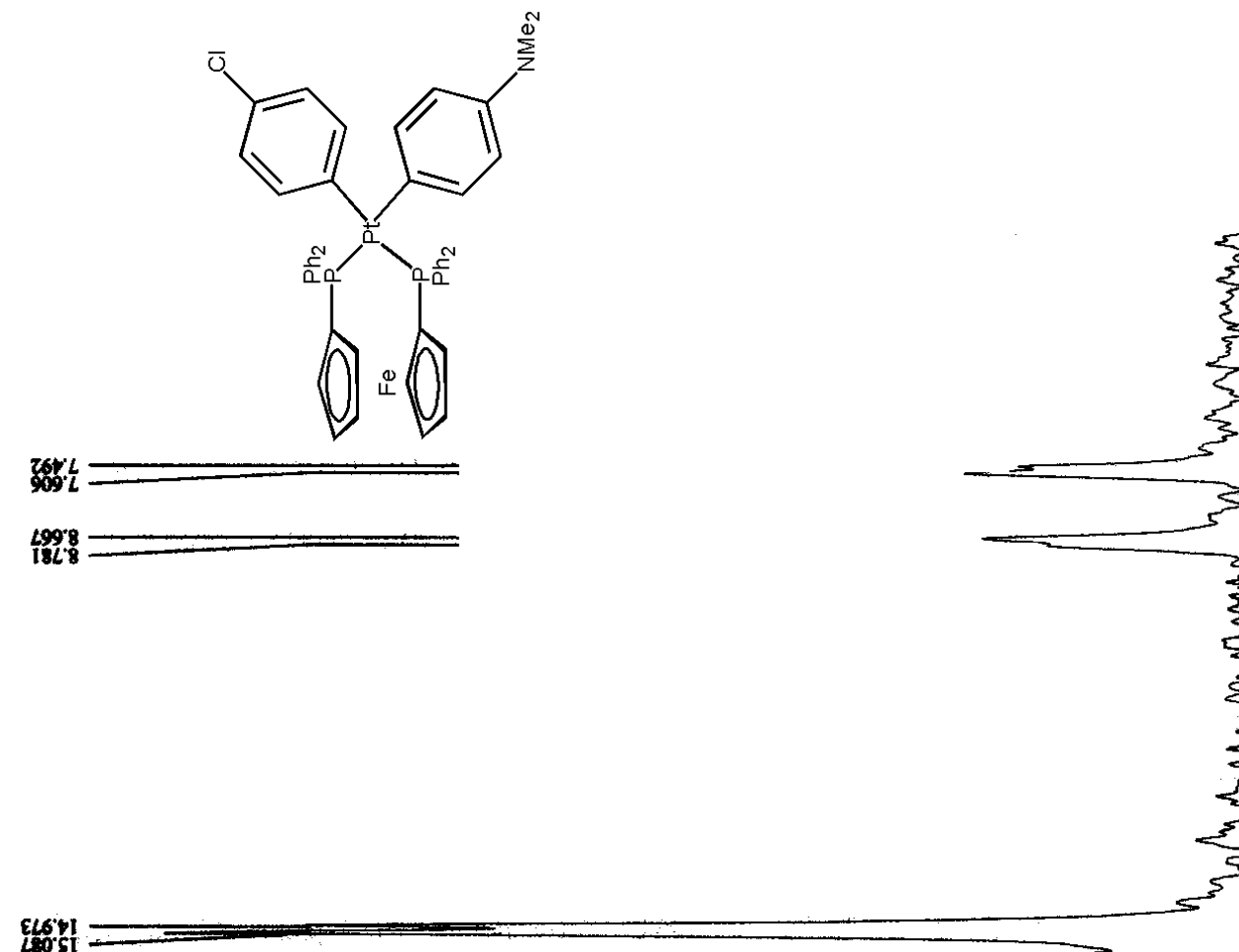

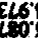
616 6091

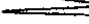

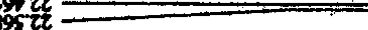

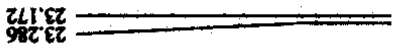

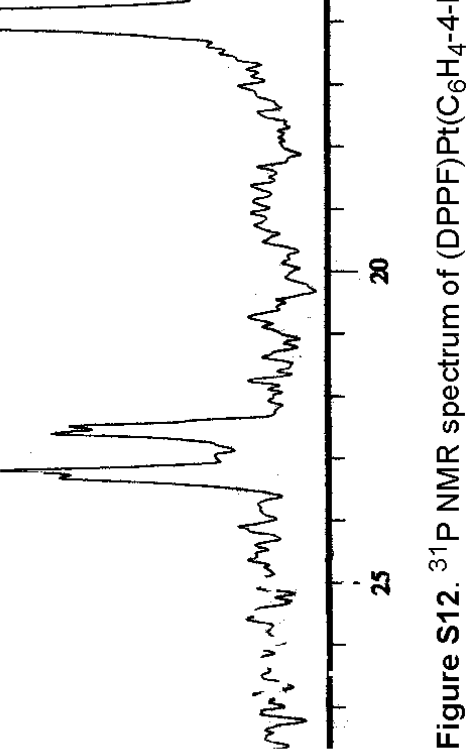



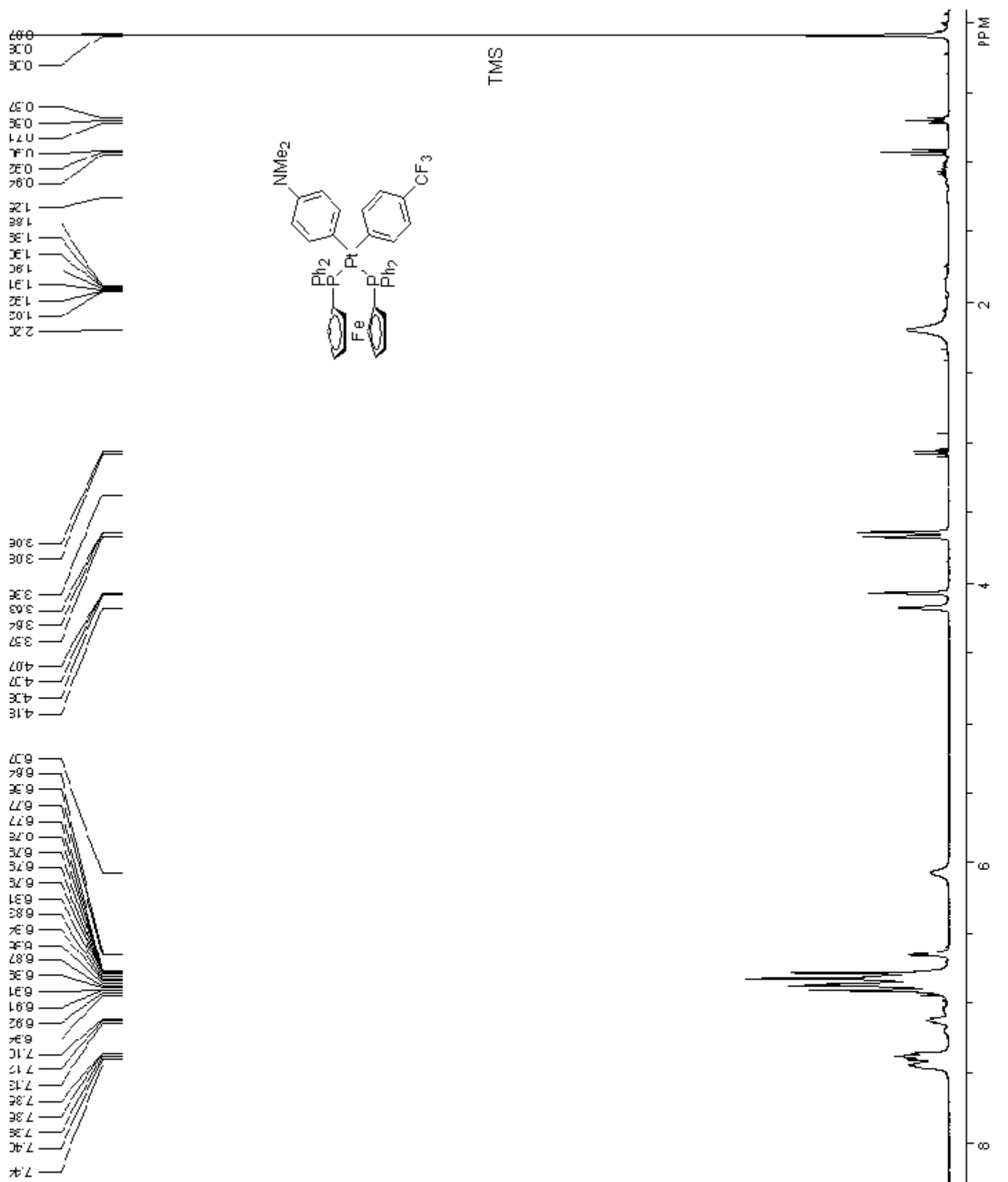

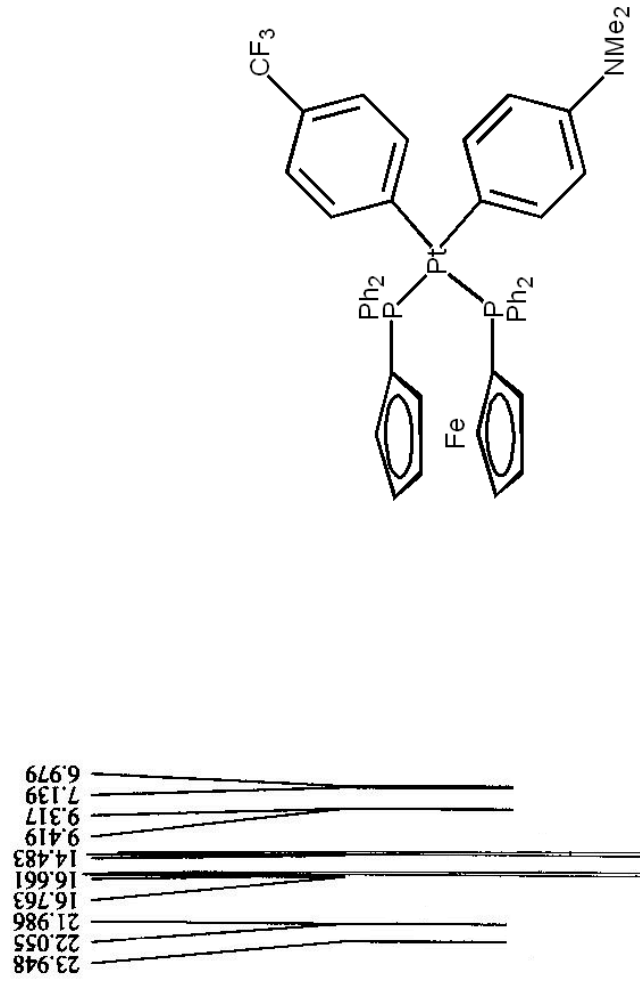

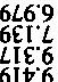

$875 \mathrm{I}$

I99.91

986.12
sso $z 2$
$816 \mathrm{EZ}$

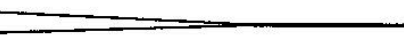

- 


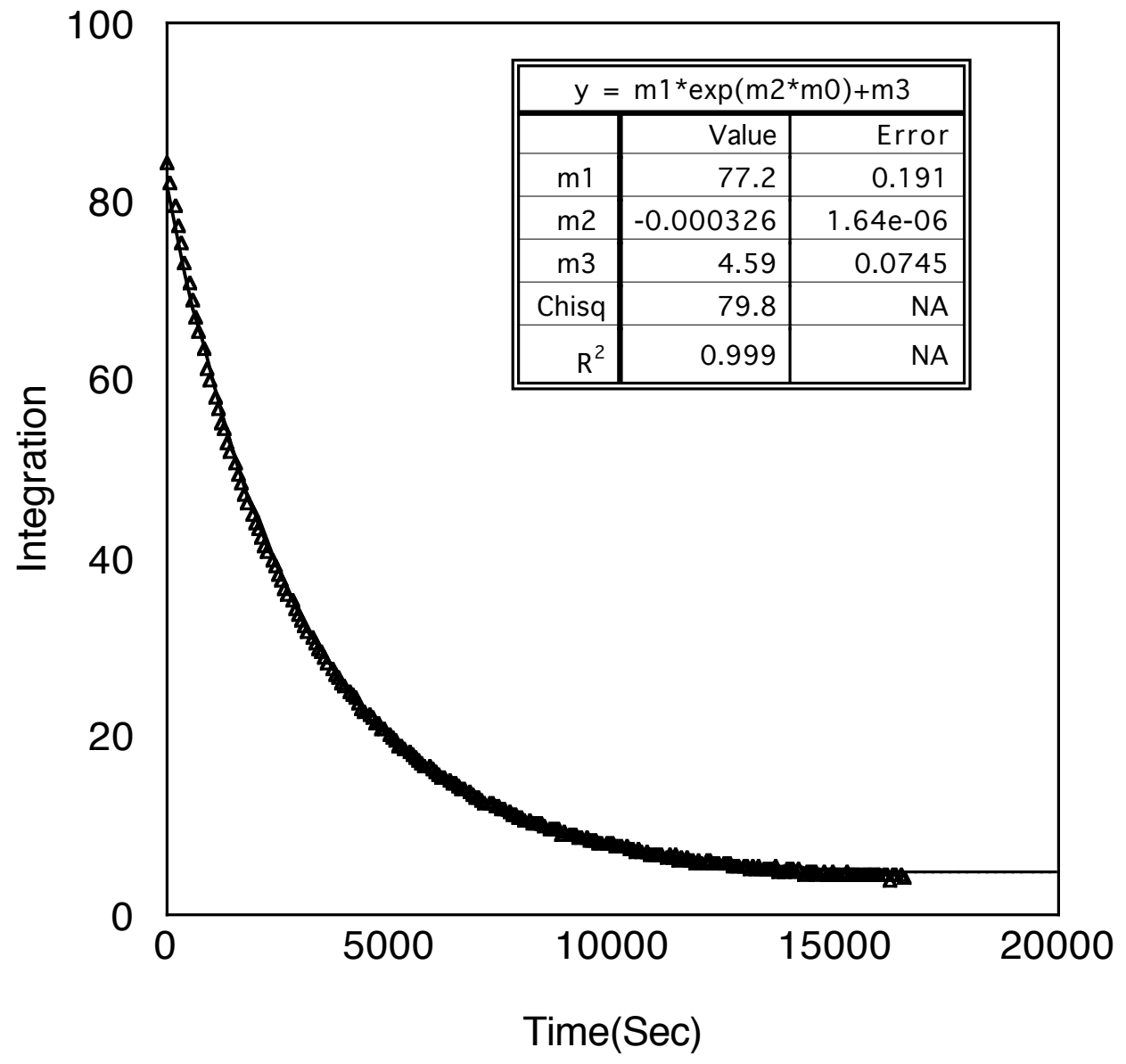

Figure S15. Plot of decay of (DPPF)Pt $\left(\mathrm{C}_{6} \mathrm{H}_{4}-4-\mathrm{NMe}_{2}\right)\left(\mathrm{C}_{6} \mathrm{H}_{4}-4-\mathrm{OMe}\right)$ at $95^{\circ} \mathrm{C}$. 